\title{
Andreas Fisahn
}

\section{Den Stier das Tanzen lehren? Europa vor neuen Herausforderungen}

\section{Krise der Demokratie - Das Offensichtliche}

Es ist mittlerweile schon ein Langweiler festzustellen, dass sich die Europäische Union in der Krise befindet. Und inzwischen hat der kritische Teil der Öffentlichkeit auch bemerkt, dass es sich nicht nur um eine im weiteren Sinne ökonomische Krise, sondern fundamental auch um eine demokratische Krise handelt. Um das aufzuzeigen genügt es, sich wenige Meilensteine der jüngsten Entwicklung in Erinnerung zu rufen. Erinnert sei zunächst an den Vorschlag des griechischen Ministerpräsidenten Andreas Papandreou vom 31.10.2011, ein Referendum über die von der EU verordnete Kürzungspolitik durchzuführen. Daraufhin ging ein Aufschrei durch die politischen Eliten der EU, besonders die deutschen. Man könne doch das Volk nicht über derart essenzielle Dinge abstimmen lassen der erste Offenbarungseid der „Demokraten“. Papandreou nützte es nichts, den Vorschlag zurückzuziehen, er musste auf Druck der EU zurücktreten und sein Kabinett wurde durch eine „Expertenregierung“ ersetzt. Weil das so gut funktioniert hatte, wurde Berlusconi gleich mitentsorgt und auch Italien erhielt mit der Regierung Monti eine „Expertenregierung“. Diese Expertenregierungen betreiben genau die gleiche Politik, wie die letzten „Expertenregierungen“ in Deutschland, die Regierungen von Papen und Schleicher, die mit ihrer Kürzungspolitik das Ende der Weimarer Republik zumindest beschleunigten. Als in Griechenland im Mai 2012 schließlich Neuwahlen stattfanden, brachten sie nicht das gewünschte Ergebnis, und die Kürzungsparteien erhielten keine ausreichende Mehrheit, um die Anordnungen der EU durchzusetzen. Wenn das Volk anders abstimmt, als es sich die politischen Eliten der EU wünschen, wird noch mal abgestimmt. Das geschah nach den gescheiterten Referenden in Dänemark und Irland; oder die Abstimmung wird vermieden, so in Holland und Frankreich. Folgerichtig ließ man in Griechenland noch einmal wählen, damit das Ergebnis stimmt. Auf den Punkt brachte die jüngste Entwicklung der CDU-Fraktionsvorsitzende Volker Kauder mit seinem Ausruf: „Jetzt wird in Europa Deutsch gesprochen.“

Die offensichtlichen Probleme haben ihre Ursache - diese These soll hier entwickelt werden - in der polit-ökonomischen Struktur der Europäischen Uni- 
on, die nicht durch einzelne Maßnahmen, schon gar nicht durch sog. Anpassungsprogramme gelöst werden können, sondern einer grundlegenden Revision der Gesamtstruktur bedürfen. Das heißt: Europa muss neu begründet werden (Bsirske u.a. 2012); die Völker Europas brauchen einen neuen Gesellschaftsvertrag. Die Alternative liegt in einer autoritären Wende oder in einer Verstärkung der zentrifugalen, d.h. national-chauvinistischen Tendenzen. Um diese These zu begründen, ist ein Blick auf die Entwicklung der EU nach dem Zweiten Weltkrieg hilfreich.

\section{Konstituierung der Zollunion}

Der europäische Bundesstaat ist keineswegs eine Erfindung der letzten Jahrzehnte. Die Diskussion um ein vereintes Europa hat viele Vorläufer mit sehr unterschiedlichen politischen Vorstellungen und Einschätzungen. Lenin schrieb im August 1915, also unter dem Eindruck des Weltkriegs, einen Aufsatz „Über die Losung der Vereinigten Staaten von Europa“, in dem er eben diese scharf ablehnte. Weil das Kapital international und monopolistisch geworden sei, könnten die Vereinigten Staaten von Europa nur dem Interesse des europäischen Monopolkapitals in der Konkurrenz zu Asien und den USA dienen und Frieden nur insofern stiften, als die Kolonien untereinander aufgeteilt werden. Ein Vereinigtes Europa könne lediglich als Abkommen darüber verstanden werden, „wie man gemeinsam den Sozialismus in Europa unterdrücken“ kann (Lenin 1970:345). Dagegen trat Trotzki acht Jahre später für ein vereinigtes Europa ein, denn: ,je schneller die Volksmassen das Vertrauen zu den eigenen Kräften zurückgewinnen, desto enger werden sie sich unter der Parole 'Union der Arbeiter- und Bauernrepubliken Europas' zusammenschließen“, wobei er meinte, „pazifistische Missdeutungen“ dieser Parole nicht befürchten zu müssen (Trotzki 1923). Trotzki lag voll daneben. Die „pazifistischen Missdeutungen“ einer Vereinigung Europas gaben den Ton an, wobei die Beseitigung der Rivalitäten zwischen den Kolonialmächten ebenso wie der "Schutz vor einer Eroberung durch Russland“ durchaus als positives Programm propagiert wurde (Coudenhove-Kalergi 1923: 20). Im Zweiten Weltkrieg nahmen auch Kommunisten allzu berechtigt die „pazifistische Missdeutung“ auf. Spinelli (PCI) und Rossi (SPI) verfassten in der Gefangenschaft Mussolinis ein Manifest für ein vereintes und freies Europa, das föderalistisch konzipiert war und das sie als ein wesentliches Element im Kampf um den Weltfrieden verstanden (Spinelli/Rossi 1941) Ähnliche Bestrebungen gab es auch im antifaschistischen Widerstand anderer europäischer Länder.

Diese sozialistisch inspirierten Ideen einer friedlichen Vereinigung Europas setzten sich allerdings nicht durch. Europa entstand aus geo- und industriepoli- 
tischen Interessen. Geopolitisch ging es um die Integration der Bundesrepublik in „den Westen“ in Abgrenzung zum Einflussbereich der UdSSR. Adenauer trieb die Westintegration der Bundesrepublik voran, was zunächst die Aufgabe der Option auf Wiedervereinigung mit der DDR bedeutete. Aus diesem Grund und aus Sorge um die nationale Souveränität Deutschlands war die EGKS wie die EWG auch in der CDU durchaus umstritten. Befürchtet wurde, dass die wirtschaftlichen Vorteile einer gemeinsamen Verwaltung der Montanindustrie nur auf französischer Seite zu finden seien (Erhard 1955).

Industriepolitisch hatte Frankreich ein großes Interesse, die kriegswichtigen deutschen Industrien, das waren damals Kohle und Stahl, zu kontrollieren und gleichzeitig auf diese Ressourcen zurückgreifen zu können. Deutschland hatte ein Interesse, die Beschränkungen der Industrieproduktion, die mit dem Ruhrstatut verbunden waren, zu beseitigen. Adenauer machte die Aufhebung des Ruhrstatuts zur Voraussetzung der Ratifikation des Vertrages über die Europäische Gemeinschaft für Kohle und Stahl (EGKS) (Adenauer 1951: 6501 f). Die EGKS ging aus dem Schumanplan hervor, benannt nach dem französischen Außenminister, der diesen von Jean Monnet entwickelten Plan am 9. Mai 1950 vorgestellt hatte. Die Idee war es, durch eine gemeinsame Verwaltung der Montanindustrie in Deutschland und Frankreich den Nukleus für eine europäische Integration zu schaffen und diese Schritt für Schritt zu erweitern. Mit der EGKS wurden für die Montanindustrie zunächst zwischen Deutschland, Frankreich, Italien und den BeNeLux-Staaten gemeinsame Regeln, Behörden, Kontrollen usw. vereinbart. Auch die Institutionen der EGKS bildeten das Urbild der Institutionen der Europäischen Wirtschaftsgemeinschaft, die mit den römischen Verträgen 1957 gegründet wurde, und später der EU.

Die SPD unter Schumacher und Ollenhauer votierte gegen die EGKS und EWG, weil sie ein Zusammenschluss der „Länder mit dem K“ sei: konservativ, klerikal, kapitalistisch, kartellistisch, während die sozialdemokratischen Länder, vor allem Skandinavien und Großbritannien, ausgeschlossen seien (Stamm 2011: LV). Den Charakter der EGKS beurteilten Zeitgenossen sehr unterschiedlich. So wurde einerseits behauptet, die EGKS sei der erste Schritt in die Kommandowirtschaft, weil die Hohe Behörde (Kommission) interventionistisch agieren könne. Auf der anderen Seite wurde kritisiert, mit der EGKS werde ein „System der Zwangskonkurrenz" geschaffen (Ritschl 1951:21), da die Montanindustrien der beteiligten Länder nicht mehr durch Zölle vor der Konkurrenz der anderen geschützt werden könnten. Mit der Zollunion war in der Tat das Fundament für eine Wettbewerbsordnung gelegt - mehr aber eben noch nicht, denn die Wettbewerbsordnung bedurfte mehr als des Fundaments. Das Gebäude auf dem Fundament wurde politisch erst mit der Einheitlichen Europäischen Akte von 1987 weiter gebaut. 
Die EGKS war aber nicht nur als Zollunion konzipiert, sie enthielt gleichzeitig starke Elemente staatsinterventionistischer und planerischer Befugnisse. Im Zweifel konnten die neuen supranationalen Organe dirigistische Maßnahmen ergreifen. So konnte die Hohe Behörde der Montanunion u.a. Preise, Erzeugungsquoten und Verwendungsprioritäten für Kohle und Stahl festsetzen. Carlo Schmid kritisierte im Bundestag für die SPD: „Nun schafft man einen europäischen Planungsapparat; aber der kann doch nur funktionieren, wenn er einen nationalen Planungsunterbau hat", was aber nicht der Fall sei (Schmid 1951: 5616). Reste dieses interventionistischen Akkumulationsmodells finden sich auf europäischer Ebene bis heute bei der Regulierung des Agrarsektors. Dort gibt es bis heute Produktionsquoten, die unterschiedlich begründet werden - beim Fischfang etwa mit Gesichtspunkten der Nachhaltigkeit, bei der Milchproduktion mit Gründen der Überproduktion und Preisstabilität.

Der EWG-Vertrag von 1957 erweiterte die Zollunion über Kohle und Stahl hinaus auf die gesamte wirtschaftliche Produktion der zunächst sechs Mitgliedstaaten. Zollunion bedeutete, dass die Mitgliedstaaten untereinander Aus- und Einfuhrzölle abbauen und nach außen gemeinsame Zolltarife entwickeln sollten. Die Römischen Verträge enthielten darüber hinaus schon die sog. vier Grundfreiheiten, also Warenverkehrs-, Kapitalverkehrs-, Dienstleistungsfreiheit und Arbeitnehmerfreizügigkeit. Die ersten drei werden noch genauer zu betrachten sein. Die Arbeitnehmerfreizügigkeit steht, verglichen mit den anderen, bekanntlich eher auf dem Papier. Der EWG Vertrag enthielt Regelungen zur Konjunkturpolitik mit einer entsprechenden Ermächtigung für die europäischen Institutionen, konjunkturpolitische Maßnahmen zu ergreifen (Art. 103 EWG 1957). In den aktuell geltenden Lissaboner Verträgen findet sich nicht einmal mehr das Wort „Konjunktur“, geschweige denn eine Kompetenznorm für eine gemeinschaftliche Konjunkturpolitik. Entsprechend problematisch war die Reaktion der EU auf die Krise von 2008. Man kann so von „einer begrenzten Öffnung der nationalen Wirtschaftsräume“ sprechen, die dazu beitrug, „die nationalen fordistischen Entwicklungspfade“ abzusichern (Bieling 2003: 45).

\section{Von der Zollunion zum Wettbewerbsstaat}

Die Römischen Verträge normierten in den Bestimmungen über die Warenverkehrsfreiheit, dass mengenmäßige Einfuhrbestimmungen sowie alle Maßnahmen gleicher Wirkung zwischen den Mitgliedstaaten verboten seien (Art. 30 EWG 1957). Diese Grundregel findet sich bis heute im Primärrecht der EU (Art. 34 AEUV), wenngleich das Verbot heute kategorischer formuliert ist, weil (zeitliche) Einschränkungen weggefallen sind. Mengenmäßige Beschränkung meint im 
Ursprung die Kontingentierung von Warenexporten oder -importen. Diese Norm schlummerte bis Ende der 1970er Jahre weitgehend unbeachtet vor sich hin, bis sie vom EuGH aus dem Dornröschenschlaf gerissen wurde. In der sog. Dassonville-Entscheidung (EuGH 1974: Rs. 8/74) subsumierte das Gericht weitgehend alle nationalen Produktregelungen, welchen Zweck sie auch immer verfolgten, der „Maßnahme gleicher Wirkung“, die ebenso wie eine mengenmäßige Einfuhrbeschränkung nach den Verträgen verboten sei. Das wurde in späteren Entscheidungen (EuGH 1979: Rs. 120/78) relativiert und bestimmte Regulierungsgründe wie etwa die menschliche Gesundheit für zulässig erklärt (Haltern 2007: \$14). Der EuGH betätigte sich euphemistisch gesprochen als „Motor der Integration“, umgekehrt lässt sich diese Rechtsprechung als nicht legitimierte Rechtsfortbildung kritisieren. An eine derart extensive Auslegung der Warenverkehrsfreiheit war bei Abschluss der Römischen Verträge sicher nicht gedacht worden. Die deutsche Politik hat zweifellos nicht intendiert, dass dereinst das Verbot „mengenmäßiger Beschränkungen“ dazu herhalten könnte, das uralte Reinheitsgebot für deutsches Bier als europarechtswidrig zu kippen (EuGH 1987: Rs. 178/84). Mit dieser Rechtsprechung hat der EuGH den Startschuss in eine neue neoliberale Konkurrenzordnung gegeben, den die Politik zum Laufen veranlasste. Startschuss bedeutet natürlich nicht, dass die Richter bewusst eine Entwicklung auf den Weg brachten oder gar deren Ergebnis, die Änderung der gesellschaftlichen Beziehungen, verstanden als politische, soziale, ökonomische und industrielle Beziehungen, im vorhinein planten oder auch nur ahnten, wie die Entwicklung verlaufen könnte. Und selbstverständlich hätte diese Entscheidung politisch zurückgeholt werden können - das Gegenteil war aber der Fall: die Politik griff den Faden auf: Mit der Einheitlichen Europäischen Akte wurde der Weg in den europäischen Binnenmarkt beschritten.

Zunächst gilt es aber, die Bedeutung der Rechtsprechung des EuGH zu erfassen. Das Gericht wurde damit zu einem der Akteure, der auf die Krise des fordistischen Modells in den 1970er Jahren reagierte: Diese Krise hatte verschiedene Dimensionen: sie stellte bereits eine ökologische Krise dar - die Nutzung der Umwelt hatte deutlicher als heute spürbar negative Effekte für die Lebensbedingungen der Menschen. In den 1970er Jahren wurden elementare Grundsteine der Umweltgesetzgebung gelegt, die bis heute wirken. Die Krise hatte aber auch eine ökonomische Dimension, die als Scheitern Keynesianischer Krisenpolitik und damit der Steuerbarkeit der Ökonomie reflektiert wurde - tatsächlich als Überakkumulationskrise die Verwertungsbedingungen des Kapitals in der alten Form industrieller Produktion verschlechterte und so die Suche nach neuen Anlagemöglichkeiten eröffnete. Und es handelte sich schließlich - deutlich sichtbar - um eine ideologische Krise oder Hegemoniekrise, die „Legitimationsprobleme im Spätkapitalismus" (Habermas 1973) erhoffen und auf der anderen Seite 
befürchten ließ. Der Weg in eine Europäische Wettbewerbsordnung war im Ergebnis eine Antwort auf alle drei Dimensionen der Krise - der Auftakt des EuGH der Beginn einer Suchbewegung.

Verschiedene Anläufe zur Veränderung der Struktur der EWG verliefen im Sande, nur das Parlament wurde, was schon in den Römischen Verträgen versprochen war, aufgewertet und 1979 erstmalig direkt gewählt. Verbunden wurde mit der Direktwahl die Hoffnung auf eine umfassende Parlamentarisierung der Europäischen Gemeinschaft, die aber nur sehr langsam voranschritt. Bis heute erfüllt das EU-Parlament nicht die Anforderungen an eine parlamentarische Demokratie, weil es noch kein regional gleiches Wahlrecht gibt, denn die Stimmen der Wahlberechtigten haben ein sehr unterschiedliches Gewicht. Mit der Einheitlichen Europäischen Akte, die 1987 in Kraft trat, wurde der Weg in den Binnenmarkt als Ziel angesteuert. Die Überwindung der Fragmentierung der europäischen Wirtschaft durch einen gemeinsamen Wirtschaftsraum sollte das Gewicht Europas in der internationalen Konkurrenz stärken. Um diese neue Wettbewerbsfähigkeit zu erreichen, die bis heute die Rhetorik der Union dominiert, galten Deregulierung und Liberalisierung als probates Mittel (Pollak/ Slomski 2012: 35). Mit ihrer Hilfe sollten "Handelshemmnisse" innerhalb der Union abgebaut werden, um so ein System zu errichten, das den Wettbewerb innerhalb des gemeinsamen Marktes vor Verfälschung schützt (Borchard 2002: 19; vgl. Art. 32, 49, 101, 107, 116, 348, AEUV).

Dabei stand die Deregulierung und Liberalisierung der Kapitalmärkte noch nicht auf der Tagesordnung der Politik, obwohl mit dem Zusammenbruch des Systems von Bretton Woods schon eine Teilglobalisierung der Finanzmärkte stattfand. Diese bezog sich zunächst auf den Devisenmarkt. In den 1980er Jahren entstand dann ein globalisierter Markt für staatliche Schuldpapiere, der sich erst in den 1990er Jahren auf die Unternehmensfinanzierung über Kapitalmärkte ausweitete (Grahl 2003: 21 ff). Damit korrespondiert das europäische Ausbuchstabieren der Kapitalverkehrsfreiheit. Diese bestimmte die Agenda der Politik, als der Schritt in die Wirtschafts- und Währungsunion beschlossen wurde. Auf der Grundlage eines Berichts von Jaques Delors wurde ein Drei-Stufen-Plan beschlossen. Die erste Stufe begann 1990 mit einer Kapitalverkehrsliberalisierung und einer verstärkten Koordinierung der Währungspolitiken, es folgte 1994 die Errichtung des Europäischen Zentralbanksystems und nach einer vorübergehenden Fixierung der Wechselkurse die Einführung des Euros am 1. Januar 2002 (Weidenfeld 2011: 82 f). Im Aktionsplan für Finanzdienstleistungen entwickelte die EU Kommission ihre Vorstellungen eines Finanzbinnenmarktes, der durch eine Liberalisierung des Kapitalverkehrsregeln herzustellen sei (EU Kommission 1999) Vertraglich fixiert wurde der Weg in die Währungsunion durch den Vertrag von Maastricht, der im November 1993 in Kraft trat. 
Nach mehreren Vertragsänderungen, die keine entscheidenden Veränderungen brachten (Amsterdam 1997, Nizza 2001), sollte schließlich eine Europäische Verfassung verabschiedet werden, mit der die Union zwar keine neue Struktur erhalten sollte, aber eine stabile Grundordnung und Entscheidungsstrukturen, die über Mehrheitsentscheidungen die komplizierten Abstimmungen nach der Osterweiterung und des Anwachsens der EU auf 27 Staaten vereinfachen sollten. Die Europäische Verfassung scheiterte durch Referenden in Frankreich und den Niederlanden. Verabschiedet wurde schließlich der geltende Lissabonner Vertrag, bestehend aus EUV und AEUV, mit dem der Text des Verfassungsentwurfes eins zu eins übernommen wurde, aber Volksabstimmungen vermieden werden konnten.

Mit dem Lissabonner Vertrag wurde die neoliberale Struktur der Union festgeschrieben und voll entwickelt. Der Binnenmarkt wird konzipiert als Wettbewerbsordnung, in der zunächst die Unternehmen der Mitgliedstaaten konkurrieren sollen, weshalb die Wirtschaftspolitik „im Einklang mit dem Grundsatz einer offenen Marktwirtschaft mit freiem Wettbewerb, wodurch ein effizienter Einsatz der Ressourcen gefördert wird," stehen soll (Art. 119, 120,127 AEUV, zur Kritik: Oberndorfer, 2011: 8). Dieses Prinzip wird dann in den Verträgen ausbuchstabiert, neben den Grundfreiheiten beispielsweise über das Subventionsverbot (Art. 107 AEUV), das die staatliche Industrie- und Regionalpolitik grundlegend verändert hat, die einseitige Festlegung der Währungspolitik auf Preisstabilität (Art. 127 AEUV) - während die ausgeglichene Handelsbilanz und die niedrige Arbeitslosigkeit nicht vorkommen -, die geforderte Privatisierung infrastruktureller Netze (Art. 170 AEUV) oder auch Kartellregelungen.

Der Binnenmarkt ohne Wettbewerbsverzerrungen geht über den Abbau der Zollschranken und Einfuhrbeschränkungen hinaus und folgt dem Prinzip der Nichtdiskriminierung. Der Wettbewerb zwischen den Unternehmen der Mitgliedstaaten schlägt um in einen Wettbewerb zwischen den Staaten, der als Standortkonkurrenz die politische Diskussion bestimmt. Die Standortkonkurrenz ist keineswegs lediglich spezifisch konservative Ideologie, die insbesondere die Regierung Kohl proklamierte, sondern entspringt den Strukturen der europäischen Wettbewerbspolitik. Wenn nämlich nationale Regulierungen der Produkte und der Produktion als diskriminierende Faktoren im Rahmen der Warenverkehrsfreiheit geschleift werden, heißt das nicht, dass sie ersatzlos wegfallen. Der Wettbewerb braucht gleiche Rahmenbedingungen; konkurriert werden soll um die ökonomische Effizienz, was vergleichbare Startbedingungen und Parcours voraussetzt. Deshalb hat sich die Europäische Union daran gemacht, Rechtsvorschriften im Bereich des Kapital- und Warenverkehrs zu harmonisieren, für alle Mitgliedstaaten einen annähernd gleichen Rechtsrahmen für den „freien“ Waren- und Kapitalverkehr zu schaffen. 
Sichtbare oder erlebbare Beispiele für diese Harmonisierung sind Regelungen, die Qualitätsanforderungen an Waren normieren. Das geschieht aus unterschiedlichen Gründen: im Lebensmittelrecht etwa, um Gesundheitsschutz zu gewährleisten, in anderen Bereichen, um Kompatibilität zwischen verschiedenen Produkten herzustellen, beispielsweise zwischen deutschen Steckdosen und französischen Steckern, was erst den „unverzerrten Wettbewerb“ zwischen den Produzenten ermöglicht. Die gelben Scheinwerfer französischer Autos verschwanden; die EU arbeitet daran, die Spurweiten für Eisenbahnen anzugleichen, um die Interoperabilität des europäischen Streckennetzes zum Zwecke des Wettbewerbs herzustellen. Schließlich verstieg sich die EU dazu, Normen etwa für Gurken, Bananen und Äpfel oder die Beschaffenheit einer Pizza zu beschließen, die teilweise wieder abgeschafft wurden. Kurz: Zum Zwecke der Herstellung eines europäischen Binnenmarktes werden die Rechtsordnungen der Mitgliedstaaten über das Europarecht harmonisiert.

Im Bereich des Warenverkehrs geschieht dies meist als Vollharmonisierung, d.h. die Mitgliedstaaten haben keine Abweichmöglichkeiten. Mindeststandards wurden dagegen für das Umweltrecht gesetzt, was es den Mitgliedstaaten erlaubt, schärfere Regelungen einzuführen oder beizubehalten. Inzwischen ist das gesamte Umweltrecht europarechtlich überformt oder besser gesagt: Deutschland ist es gelungen, die meisten seiner umweltrechtlichen Regelungen vom Abfall- über das Immissionsschutz- bis zum Naturschutzrecht nach Europa zu exportieren. D.h., die europarechtlichen Regelungen lehnen sich stark an die älteren deutschen Regelungen an. So ist ein - verglichen mit den 1950er bis 70er Jahren oder anderen Regionen der Welt - recht effektives europäisches Umweltregime geschaffen worden. Das ging über die europäische Union besser als über nationalstaatliche Regelungen, weil die Konkurrenz der Industrien um die Umweltnutzung jedenfalls auf europäischer Ebene dem Interesse des ideellen Gesamtkapitalisten, der ja keineswegs ein Interesse an einer zerstörten Umwelt hat, untergeordnet wurde.

\section{Dimensionen der Krisenlösung}

Damit wurde die erste Dimension der Krise der 1970er Jahre eingedämmt, was nicht bedeutet, dass es umweltpolitisch keine Probleme mehr gibt - aber die Fortschritte etwa bei der Wasser- und Luftreinhaltung sollte man nicht ignorieren. Die ökologische Krise hat sich gleichsam verlagert von der nationalen und regionalen auf die globale Ebene, denn peak oil und der Klimawandel sind keine regionalen Probleme und in einer einzelnen Weltregion nicht mehr zu lösen. Zweitens wurden mit dem Binnenmarkt die rechtlichen Grundlagen geschaffen für eine Krisenlösung über verstärkten Außenhandel. Dabei ist der Freihandel 
nicht auf Europa begrenzt und findet seine globale Dimension im rechtlichen Regime der WTO. Dennoch ist der europäische Binnenmarkt für die Überwindung der Verwertungskrise der 1970er Jahre bis heute relevant, denn ein Großteil der Warenexporte etwa der BRD entfällt auf den innereuropäischen Handel. Das Kapital suchte in der Krise die Lösung über die Ausweitung der Absatzmärkte. Dafür wurden über das rechtliche und institutionelle Arrangement der Europäischen Union die Restriktionen des Außenhandels abgebaut. Dies lässt sich sogar als bewusste Strategie identifizieren, die von der zusammenfassend als neoliberal charakterisierten Wirtschaftswissenschaft propagiert wurde, deren Marktradikalismus auch deshalb auf fruchtbaren Boden fallen konnte, weil der sozialdemokratische Interventionsstaat gleichsam in einer Zangenbewegung von links und rechts angegriffen wurde.

Damit rückt die dritte Dimenion der Krise der 1970er Jahre, die Krise der Ideologie und Hegemonie, in den Blick. Um die Verschiebungen zu erfassen, die mit der Europäisierung stattgefunden haben, sind zwei Analysen des bürgerlich, parlamentarischen Verfassungsstaates in Erinnerung zu rufen. Schon Marx hat den grundlegenden Widerspruch der bürgerlich demokratischen Verfassung so analysiert: „Die Klassen, deren gesellschaftliche Sklaverei sie verewigen soll, Proletariat, Bauern, Kleinbürger, setzt sie durch das allgemeine Stimmrecht in den Besitz der politischen Macht. Und der Klasse, deren alte gesellschaftliche Macht sie sanktioniert, der Bourgeoisie, entzieht sie die politischen Garantien dieser Macht. Sie zwängt ihre politische Herrschaft in demokratische Bedingungen, die jeden Augenblick den feindlichen Klassen zum Sieg verhelfen und die Grundlagen der bürgerlichen Gesellschaft selbst in Frage stellen. Von den einen verlangt sie, dass sie von der politischen Emanzipation nicht zur sozialen fort-, von den anderen, dass sie von der sozialen Restauration nicht zur politischen zurückgehen“ (Marx 1850: 43). Dieser Widerspruch wurde in der Geschichte der kapitalistischen Gesellschaft auf unterschiedlichen Wegen zugunsten der gesellschaftlich mächtigen Klassen gelöst oder zu lösen versucht: angefangen vom Klassenwahlrecht mit unterschiedlichem Stimmgewicht oder gut organisiertem Mehrheitswahlrecht (Canfora 2006), über Mechanismen der ideologischen und sozialen Integration bis zu autoritären oder faschistischen Regimen (Fisahn 2008: $94 \mathrm{ff}$ ). Die Verfassung lässt sich deshalb verstehen als Kompromiss, auf den sich die gesellschaftlichen Klassen einigen, auf dessen Grundlage die gesellschaftlichen Auseinandersetzungen in Zukunft ausgetragen werden und der gleichzeitig die Kräfteverhältnisse fixiert (Marx 1850: 41; Abendroth 1979: 255). Abendroth verstand das Grundgesetz (GG) als Klassenkompromiss, der sich charakterisieren lässt durch die Formel: Sozialstaat und kollektive Grundrechte gegen Verzicht auf Enteignung, soziale Grundrechte und sozialistische Wirtschaft. So blieb das GG wirtschaftspolitisch neutral, was impliziert, dass über die wirtschaft- 
liche Ausrichtung der demokratische Prozess entscheidet. So formulierte das Bundesverfassungsgericht: „Das Grundgesetz garantiert weder die wirtschaftspolitische Neutralität der Regierungs- und Gesetzgebungsgewalt noch eine nur mit marktkonformen Mitteln zu steuernde 'soziale Marktwirtschaft'. Die 'wirtschaftspolitische Neutralität' des Grundgesetzes besteht lediglich darin, dass sich der Verfassungsgeber nicht ausdrücklich für ein bestimmtes Wirtschaftssystem entschieden hat. ... Die gegenwärtige Wirtschafts- und Sozialordnung ist zwar eine nach dem Grundgesetz mögliche Ordnung, keineswegs aber die allein mögliche. Sie beruht auf einer vom Willen des Gesetzgebers getragenen wirtschafts- und sozialpolitischen Entscheidung, die durch eine andere Entscheidung ersetzt oder durchbrochen werden kann" (BVerfGE 4, 7 [17 f]).

Mit der europäischen Konstitution, wie sie in den Lissabonner Verträgen fixiert wurde, ist diese Kompromissstruktur entscheidend verschoben worden. Erstens wurde die offene Marktwirtschaft festgeschrieben und damit nicht nur der Weg in ein anderes Wirtschaftssystem versperrt. Darüber hinaus wurde eine - im Vergleich zu den interventionistischen, sozialstaatlichen Konzeptionen, die bei Gründung von EGKS und EWG Pate standen - marktradikale oder neoliberale Ordnung festgeschrieben. Die Wirtschaftsordnung wurde so inhaltlich der demokratischen Gestaltung entzogen. Die nationalen Regierungen werden zweitens im Wettbewerb sensibler für die Imperative des Marktes als für die des demokratischen Prozesses (Gill 1998: 5). Drittens wurde diese neoliberale Ordnung auch formal der demokratischen Gestaltung entzogen, indem die Entscheidungskompetenzen auf die Europäische Exekutive, also die Kommission und den Rat, verlagert wurden, während das Europäische Parlament demokratischen Ansprüchen gleicher Repräsentation immer noch nicht genügt. Wichtiger ist, dass weiter in wichtigen Politikfeldern ohne Zustimmung des Parlaments entschieden werden kann und die informellen Verhandlungspositionen, die den Parlamentsbetrieb in den Nationalstaaten kennzeichnen, wegfallen, weil die Kommission mehr oder weniger autonom agieren kann. Schließlich funktioniert der Willensbildungsprozess in der EU mittelbar über die Regierungen, womit eine weitere Schleuse gegen die direkte Einflussnahme der unteren Klassen auf die politische Entscheidungsfindung eingebaut ist (Buckel u.a. 2012: 15), was diffus durchaus bewusst zu sein scheint und sich in Wahlenthaltungen äußert. Die Europäisierung ist somit auch als Antwort auf die Hegemoniekrise der 1970er Jahre zu verstehen.

Die Krisenlösung produziert aber neue Probleme. Nehmen wir als Ausgangspunkt das Umweltrecht und die Folgen der Harmonisierung: Die Nutzung „kostenloser" Umweltgüter oder die „kostenlose“ Zerstörung der Umwelt bei der Produktion sind ein nicht zu unterschätzender Faktor im Wettbewerb. Das heißt aus der Perspektive des Einzelkapitals geht es darum, sich eine möglichst kosten- 
lose Nutzung der natürlichen Umwelt zu sichern oder jedenfalls sicherzustellen, dass für andere die Nutzung nicht billiger ist. Der Wettbewerb findet also um die mit Blick auf die Umweltkosten günstigeren Produktionsstandorte statt. Bei einer weitgehenden Harmonisierung in Europa entfällt dieser Wettbewerb. Niedrigere Umweltstandards können erstens keinen Standortvorteil für die Unternehmen mehr bringen, und auch die Staaten können zweitens diese Karte nicht mehr spielen, also niedrige Umweltstandards als Standortvorteil „vermarkten“.

Verallgemeinert man diesen Gedanken, dann findet die Standortkonkurrenz der Staaten in den Bereichen statt, in denen es keine europäische Harmonisierung gibt. Und diese Politikbereiche sind vor allem Steuern und Soziales. Im Bereich des Steuerrechts hat die EU nach Art. 113 AEUV eine Kompetenz nur für indirekte Steuern, und dies nur unter der Voraussetzung, dass solche Steuern für das Funktionieren des gemeinsamen Marktes „notwendig sind“ und der Rat einen einstimmigen Beschluss fassen kann. Im Feld der Sozialpolitik hat die EU durchaus Kompetenzen vor allem im Bereich der Antidiskriminierung, die - blickt man etwa auf die Gleichstellung der Geschlechter - aus deutscher Perspektive einen Schub nach vorn gebracht hat. Es fehlen aber Kompetenzen für eine Politik der sozialen Sicherungssysteme. So entsteht im Standortwettbewerb der Mitgliedstaaten notwendig ein Druck auf diese Politikfelder, der sich in einem „race to the bottom" aufSeiten der sozialstaatlich organisierten Mitgliedstaaten verwirklicht (Huffschmid 2008: 72).

Die Dienstleistungsfreiheit wurde von der Kommission als Vehikel eingesetzt, um einen europäischen Arbeitsmarkt in dem Sinne zu schaffen, dass Unternehmen ihre Dienstleistungen zu Tarifen des Heimatlandes anbieten. Rumänische Stahlbauer werden dann in Schweden nach rumänischen Tarifen entlohnt. Die Rechtsprechung des EuGH verstärkte diesen Effekt des Lohndumpings, indem sie Tariftreueregelungen in nationalen Gesetzen, welche die Bindung an die Tariflöhne am Ort der Arbeitsleistung vorsahen, für europarechtswidrig erklärte (EuGH 2007: Rs. C-346/05) und indem sie - in den Fällen Viking (EuGH 2007: Rs. C-438/05) und Laval (EuGH 2007: Rs. C-341/05) - das Streikrecht zu Gunsten der Europäischen Wirtschaftsfreiheiten ausgehebelt hat. Das muss zu einem innereuropäischen Lohnwettbewerb führen, der die Länder mit höheren Löhnen unter Lohndruck setzt, d.h. Lohndumping provoziert.

Folge dieses ",race to the bottom“ ist eine Umverteilung von unten nach oben, die am Beispiel der Steuereinahmen gut verdeutlicht werden kann. Während 1950 die Lohnsteuer ca. 10\% unterhalb der Einkommensteuer lag und die Körperschaftssteuer nur wenig davon abwich, hat sich bis zum Jahr 2010 das Verhältnis dieser Steuereinnahmen zueinander radikal verschoben. Die Einkommensteuer macht noch ein Viertel der Lohnsteuer aus und die Körperschaftssteuereinnahmen sind auf ein Zehntel der Lohnsteuereinahmen gesunken. Gleichzeitig waren 
2010 die Einnahmen aus der Mehrwertsteuer, die die unteren Einkommen stärker belastet als die Reichen, höher als die aus der Lohnsteuer.

Wenn der Steuerwettbewerb als Standortkonkurrenz zu einer Umverteilung von unten nach oben führt, hat dies zwei Konsequenzen. Erstens brechen den Staatshaushalten die Einnahmen weg. Auch das lässt sich statistisch belegen: Die Abgabenquote sank im Euroraum von 41,2 \% im Jahre 2000 auf 39,7 \% im Jahre 2008. Ähnlich sehen die Zahlen für Deutschland aus: die Quote fiel im gleichen Zeitraum von 41,9 \% auf 39,3\%. Der Spitzensteuersatz für Einkommens- und Körperschaftssteuer sank zwischen 2000 und 2008 im Euroraum von 48,4\% auf 42,4\%. Die höchsten Einnahmerückgänge bei der Einkommens- und Körperschaftssteuer sind zwischen 2000 und 2008 in Bulgarien (von 32,5\% auf 10,0\%), Deutschland (von 51,6\% auf 29,8\%), Zypern (von 29,0\% auf 10,0\%) und Griechenland (von 40,0\% auf 24,0\%) zu verzeichnen (Eurostat 2010). Die Verschuldung der Staaten, mit der sich die europäischen Regierungen aktuell herumschlagen, ist sicher nicht nur, aber auch auf diesen Wettbewerb um niedrige Steuern für Unternehmen zurückzuführen. Zweitens bewirkt die Umverteilung nach oben - neben anderen Faktoren -, dass Kapital akkumuliert wird, das nach neuen Anlagemöglichkeiten und günstigen Verwertungsbedingungen sucht. Es fand sie auf den Finanzmärkten mit hohen Gewinnmargen im Spekulationsgeschäft. Diese Form der Verwertung setzt aber entsprechende rechtliche Rahmenbedingungen voraus, welche die EU in den 1990er Jahren ausbuchstabierte.

\section{Durch die Kapitalverkehrsfreiheit zum europäischen Kasino}

Im Bereich der Kapitalverkehrsfreiheit wurde durch Deregulierung harmonisiert und damit den Vorgaben der Verträge gefolgt. Auch hier ist ein Vergleich der geltenden Vorschriften mit denen der Römischen Verträge aufschlussreich. Nach dem EWG-Vertrag von 1957 sollten die Mitgliedstaaten Beschränkungen des Kapitalverkehrs untereinander beseitigen - und dann folgt die Einschränkung: „soweit es für das Funktionieren des Gemeinsamen Marktes notwendig ist“ (Art. 67). Die allgemeine Wirtschafts- und Währungspolitik war in den Verträgen von 1957 lediglich als „Angelegenheit gemeinsamen Interesses“ eingestuft, die nicht durch gemeinsame Rechtsmaßnahmen, sondern lediglich in Form von „Empfehlungen an die Mitgliedstaaten“ koordiniert werden sollte (Müller 2007). Der entscheidende Punkt ist, dass die Liberalisierung des Kapitalverkehrs unter den Erforderlichkeitsvorbehalt gestellt wird, was den Mitgliedstaaten gestattete, Beschränkungen des Kapitalverkehrs beizubehalten. Das eingeschränkte und bedingte Postulat, diesen zu liberalisieren, galt bis zum Maastricht Vertrag 1993. 
Deutschland kannte unter dem Regime der Römischen Verträge unterschiedliche Formen der Finanzmarktregulation, die zu unterschiedlichen Zeiten oder nebeneinander existierten und z.T. abgeschafft und wieder eingeführt wurden. So gab es eine Genehmigungspflicht für die Verzinsung von Einlagen auf ausländischen Konten. Das Wertpapiergeschäft war in unterschiedlicher Intensität genehmigungspflichtig, wie etwa der Erwerb inländischer Wertpapiere durch Gebietsfremde. Das war verbunden mit einer Kapitalertragssteuer (Kuponsteuer) für Gebietsfremde, die inländische Wertpapiere erwarben. Sog. Pensionsgeschäfte mit Gebietsfremden ${ }^{1}$ waren ebenso genehmigungspflichtig wie der Verkauf inländischer Wertpapiere an Gebietsfremde oder die Aufnahme von Krediten im Ausland und die Veränderung der terms of payment, verstanden als vorgezogener Einzahlungen und verzögerter Auszahlungen. Es gab zeitweise eine besondere Mindestreservepflicht ${ }^{2}$ auf Einlagen Gebietsfremder und eine Bardepotpflicht, um die Aufnahme von Auslandskrediten zu verteuern (Schmidt 2007: 67 ff). Frankreich verfolgte im Devisengesetz von 1966 das Ziel einer Devisenbewirtschaftung, die mit einem umfangreichen Instrumentarium von Kapital- und Devisenkontrollen erreicht werden sollte. In Italien war die Genehmigungspflicht von Devisen- und Kapitaltransfer ins Ausland die Regel (Weniger 1988: $110 \mathrm{ff}$ ). Solche Kapitalverkehrskontrollen waren die Lehre aus der Weltwirtschaftskrise 1929 und wurden von den europäischen Staaten in der ein oder anderen Form bis in die 1990er Jahre angewendet. 1994 hat Griechenland als letzter Mitgliedstaat der Europäischen Union seine Kapitalverkehrskontrollen abgeschafft (Huffschmid 1999: 121).

Die Regelungen zur Kapitalverkehrsfreiheit im Lissabonner Vertrag (Art. 63 ff) haben die Ausnahmevorschriften der Römischen Verträge beseitigt und gehen damit weiter als die Bestimmungen zur Warenverkehrsfreiheit. Es gilt ein striktes, uneingeschränktes Verbot von Kapitalverkehrsbeschränkungen, das als „umfassende Liberalisierungspllicht" (Groeben/Schwarze 2003: Art.56, Rn.30) des Kapitalverkehrs verstanden wird. Kapitalverkehrsfreiheit normieren die EUVerträge nicht nur zwischen den Mitgliedstaaten, sondern auch in Beziehung zu Drittstaaten. So wurde ein Rückschrittsverbot bei der Liberalisierung des Kapitalverkehrs etabliert. Die Liberalisierung gegenüber Drittstaaten kann mit

1 Vermögensgegenstände wie Wertpapiere werden dabei durch einen gebietsfremden Pensionsnehmer von einem gebietsansässigen Pensionsgeber erworben, wobei sich letzterer verpflichtet, die Gegenstände wieder zurückzunehmen - sie werden beim Pensionsgeber gleichsam in Pension genommen.

2 Mindestreserven sind unverzinsliche Guthaben, die von den Geschäftsbanken bei der Zentralbank zu unterhalten sind und die in einem bestimmten prozentualen Verhältnis zu den Einlagen stehen. 
einfacher Mehrheit beschlossen, aber nur einstimmig wieder zurückgenommen werden (Art. 64 AEUV).

Der EuGH nahm das auf und beschied, dass eine nationale Regelung, nach der die Ausfuhr von Hartgeld, Banknoten oder Inhaberschecks von einer vorherigen Genehmigung abhängig gemacht wird, europarechtswidrig sei, weil sie gegen die Kapitalverkehrsfreiheit verstößt (EuGH RS-C-163/94, C-165/94 und C-250/94). In den Fällen zu den sogenannten „Goldenen Aktien“, für welche die Entscheidung zum VW Gesetz (EuGH Rs. C-282/04) nur ein Beispiel ist, dehnte der EuGH die Kapitalverkehrsfreiheit so weit aus, dass er Stimmrechtsvorteile des jeweiligen Staates grundsätzlich wegen eines Verstoßes gegen die Kapitalverkehrsfreiheit untersagte.

Die Liberalisierung des Kapitalverkehrs erreichte mit dem Investmentmodernisierungsgesetz der rot-grünen Koalition aus dem Jahre 2003 einen gewissen Höhepunkt. Veranlasst durch eine Änderung der OGAW-Richtlinie (EWG 1985) wurden in Deutschland bestimmte Geschäftspraktiken erlaubt, die typisch für Kapitalfonds sind, und deren Ausbreitung dann von Franz Müntefering als Einfall der Heuschrecken beklagt wurde. Die Gesetzesbegründung zum Investmentmodernisierungsgesetz entbehrt nicht einer gewissen Ironie: „Anbieter von Hedgefonds sollen nach dem Gesetzentwurf in Deutschland auf moderne und liberale rechtliche Rahmenbedingungen treffen. Der Finanzplatz Deutschland ist mittlerweile reif für die Zulassung von alternativen Investmentprodukten. Die Hedgefonds-Branche scheint sich zu einer Branche entwickelt zu haben, die sich der mit Hedgefonds verbundenen Risiken bewusst ist und mit ihnen verantwortungsvoll umgeht. Es darf daher erwartet werden, dass die vom Gesetzgeber vorgesehenen Freiräume nicht missbraucht werden“" (BT-Drs. 15/1553, S. 67).

Aus der konstitutionell abgesicherten Kapitalverkehrsfreiheit folgt nicht, dass es in der EU keine Regeln für die Finanzmärkte gibt. Diese sind aber im höchsten Grade marktkonform und sehen interventionistische Eingriffe in den Kapitalverkehr nicht vor. Eine nachhaltige Re-Regulation der Finanzmärkte, wie sie nach dem Finanzmarktcrash 2008 von den G20 angekündigt wurde, hat nicht stattgefunden (Fisahn 2011) und widerspricht dem Wortlaut, dem Ziel und der gegenwärtigen Auslegung der Kapitalverkehrsfreiheit im europäischen Primärrecht.

Die Re-Regulierung der Finanzmärkte wurde nicht nur deshalb zum Rohrkrepierer, weil die Interessen der Banken und anderer Spieler im globalen Kasino der Finanzwirtschaft mächtig sind. Vielmehr hat sich die Spekulation, der Vermögenseffekt der Spekulation zum Akkumulationsmodell entwickelt, das durchaus auch „realwirtschaftliche“ Effekte zeigt. Denn der gewonnene Spekulationsgewinn kann, solange das Roulette sich dreht, in der Welt der wirklichen Waren ausgegeben werden und wirkt damit auf die Konjunktur. Die Spekulati- 
onsgewinnler konsumieren Waren, die produziert werden müssen und steigern so die Nachfrage in der „Realwirtschaft“. „Die Gesellschaft wird durch fiktiven Reichtum real reicher. Leider gilt auch das Umgekehrte. Brechen Spekulation und Vermögenspreise zusammen, sackt auch die Nachfrage ... wieder in sich zusammen. Der verschwindende fiktive Reichtum löst eine Rezession aus (Zeise 2010, 160 f).

\section{Perspektiven der Entwicklung Europas}

Diese Situation ist 2008 eingetreten und seitdem agiert die europäische Union im Krisenmodus. Aus deutscher Perspektive ist aus der ökonomischen Krise eine Schuldenkrise geworden, weil sich die deutsche Ökonomie - jedenfalls vorübergehend - aufWachstumskurs befindet. Die Krisenreaktion hatte unterschiedliche Phasen. Auf die Finanzkrise antworteten die Staaten allesamt mit nationalen Bankenrettungsschirmen. In der BRD wurde der SoFFin aufgelegt mit einem Volumen von $400 \mathrm{Mrd}$. Euro Bürgschaftsermächtigung und weiteren $80 \mathrm{Mrd}$. einbezahltem Kapital (FMSF-G 2008), der ausschließlich dazu diente, deutsche Banken zu stützen. In dieser ersten Phase gab es keine europäische Bankenrettung. Die Staaten reagierten nach dem Moto „Rette sich wer kann“, womit zentrifugale Tendenzen auch im Zentrum der EU deutlich sichtbar wurden.

Auf die nationale Bankenrettung folgten ebenfalls nationale Konjunkturprogramme, die aber immerhin europäisch - und sogar global - in dem Sinne abgestimmt wurden, dass in allen Staaten Investitionsprogramme aufgelegt wurden, so dass die staatlich induzierte Nachfrage nicht einfach diffundieren konnte. Die EU verkündete, es gebe ein europäisches Konjunkturpaket in einer Größenordnung von $200 \mathrm{Mrd}$. Euro. Dieses Paket bestand jedoch aus der Summe der nationalen Pakete und weiteren $30 \mathrm{Mrd}$. ohnehin geplanten, aber vorgezogenen und umgeschichteten Investitionen der Union (EU Kommission 2008). Die EU hat keine Kompetenzen und keine Mittel, um eine eigenständige Konjunkturpolitik zu betreiben. Deutschland legte ein Konjunkturprogramm in Höhe von - im weltweiten Vergleich bescheidenen - 80 Mrd. auf.

Im dritten Akt der Krise wurde das Finanzsystem der Eurozone über Kredite an einzelne Staaten, zunächst Griechenland, Portugal, Irland und dann auch Zypern und Spanien, zu stabilisieren versucht. Kredite wurden im Frühjahr 2010 als Direkthilfen an Griechenland vergeben, das sich - ebenso wie die anderen Staaten - Kredite auf dem Finanzmarkt nur zu nicht refinanzierbaren Zinsen leihen konnte. Schon im Mai 2010 wurde für solche „Hilfsprogramme“ ein Rettungsfonds, der EFSF, eingerichtet, der Bürgschaftsgarantien in Höhe von $440 \mathrm{Mrd}$ Euro eingehen kann. Dieser Fonds soll durch einen dauerhaften Stabilitätsmechanismus (ESM) abgelöst werden, in den - anders als in den EFSF 
- ein bestimmter Anteil bar eingezahlt werden muss. Das Gesamtvolumen der Garantieermächtigung soll insgesamt bei $700 \mathrm{Mrd}$. liegen, wovon die BRD 190 Mrd. garantiert, sofern nicht Nachschusspflichten entstehen, etwa weil andere Staaten ihren Anteil nicht stemmen können.

Die „Schuldnerstaaten“ profitieren allerdings nur im geringen Umfang von den Hilfen. Von den 164 Mrd., die an Griechenland gezahlt wurden, kamen nur $21 \mathrm{Mrd}$. dem Staatshaushalt zugute. Von $79 \mathrm{Mrd}$. für Portugal gingen $24 \mathrm{Mrd}$. und von 67,5 Mrd. für Irland 15,6 Mrd. in den Staatshaushalt. Der Rest blieb im Finanzsystem (FTD 18.7.2012), d.h. stabilisierte auch das Privatbankensystem in den „Geberländern“. Im Falle Spanien sollen die Hilfen direkt an die maroden Banken fließen, was von der Regierungsmehrheit im Bundestag verbal abgelehnt wird, weil sie den Staat als Schuldner in der Pflicht wissen wollen. Was nichts anders heißt als: Der Nationalstaat haftet für „seine“ nationalen Banken.

Die „Hilfen“ dürfen zukünftig nach dem ESM-Vertrag und wurden auch bisher nur gegen rigide Kürzungs-Auflagen für die Staaten vergeben. Die Kürzungen betrafen im wesentlichen soziale Standards. Angeordnet wurden beispielsweise Kürzungen staatlicher Gehälter, eine Reduzierung des öffentlichen Dienstes auch durch Privatisierung von Bereichen öffentlicher Daseinsvorsorge, für die der AEUV nicht schon den Wettbewerb anordnet, nämlich für das Gesundheitswesen, die Bildung oder die Wasserversorgung. Angeordnet wurden Mehrwertsteuererhöhungen, die Anhebung des Rentenalters oder direkte Rentenkürzungen, eine Deregulierung des Arbeitsmarktes und schließlich eine Reduzierung der Lohnkosten (auch außerhalb des öffentlichen Sektors), was nur mit Eingriffen in die Tarifautonomie umgesetzt werden kann.

Inzwischen hat sich zumindest außerhalb Deutschlands herumgesprochen, dass die verordneten Kürzungsprogramme die ökonomische und finanzielle Misere der Staaten verschärfen und nicht etwa beheben. Die Folgen für die Länder waren verheerend, was am Beispiel Griechenland exemplifiziert werden kann. Griechenland konnte im Zeitraum zwischen 2001 und 2007 ein Wachstum des Bruttoinlandsprodukte von 28,3\% verzeichnen, das im gleichen Zeitraum für die Bundesrepublik nur 8,6\% betrug. Mit der Finanzkrise geriet ganz Europa in die Rezession. Die Wirtschaftsleistung der Bundesrepublik fiel 2009 um 4,9\%, konnte sich aber bald wieder erholen, so dass zwischen 2008 und 2010 ein Minus von 1,6\% zu konstatieren ist, 2011 wuchs die Wirtschaft der Bundesrepublik nach Angaben der EU Kommission um 2,9\%. Für Griechenland wurde zwischen 2008 und 2010 ein Minus von 6,6\% festgestellt und auch 2011 befand sich Griechenland weiter in der Rezession mit einem Minus von 5,5\% des BIP. Das hatte zur Folge, dass der Durchschnittsverdienst in Griechenland allein zwischen Mai 2011 und 2012 um ein Viertel sank, die Arbeitslosigkeit im Juni 2012 auf 21 \% anstieg und die Jugendarbeitslosigkeit von (bereits zu hohen) ca. $25 \%$ in 2008 auf 
gegenwärtig über $50 \%$ wuchs. Zu Recht wird inzwischen von einer verlorenen Generation im Süden der EU gesprochen. Diese "nackten“ Zahlen deuten auf soziale Verwerfungen hin, die für die Bevölkerung kaum noch erträglich sind.

Gleichzeitig führten die Kürzungsprogramme keineswegs zu einer Reduzierung des Haushaltsdefizits. Die Neuverschuldung stieg in Griechenland von 2,6\% im Jahre 2006 auf 12,7\% in 2009 und hatte 2011 einen Umfang von $11,9 \%$. Durch die gesunkene Wirtschaftsleistung und die erhöhte Neuverschuldung stiegen die griechischen Gesamtschulden von - das Maastricht-Kriterium deutlich überschreitenden - 97,1\% in 2006 auf 165\% des BIP im Jahre 2011 (EU Kommission 2011). Die von der EU verordnete Austeritätspolitik hatte im Ergebnis erstens eine desaströse Verschlechterung der sozialen Lage in Griechenland zur Folge und war zweitens zur Erreichung des proklamierten Zieles, nämlich Abbau der Schulden, ungeeignet.

Die Antwort auf die Krise, die von der Bundesregierung verfolgt wird, besteht prima facie aus „Sparprogrammen“. Neue Schuldenobergrenzen von nur 0,5\% des BIP wurden im Fiskalpakt vereinbart. Das kann (abgesehen von politisch nicht gewollten Steuererhöhungen) nur durch Kürzungen im Bereich der staatlichen Leistungen geschehen, d.h. durch eine verschärfte Umverteilung von unten nach oben. Um diese auch durchzusetzen, versucht insbesondere die BRD, der Europäischen Exekutive ein Durchgriffsrecht auf die nationalen Haushalte zu verschaffen. Mit dem sog. „six-pack“, einem Paket von Richtlinien und Verordnungen wurde die Berichtspflicht in Haushalts- und Wirtschaftsprogrammen verschärft. Der Fiskalvertrag verpflichtet nun Staaten, die ein übermäßiges Defizit im Sinne des Maastricht Vertrages haben, der europäischen Exekutive Haushalts- und Wirtschaftspartnerschafts(!)programme zur Genehmigung vorzulegen, d.h. die EU bekommt mit Blick auf die nationalen Haushalte eine Vetoposition. Ratspräsident Rompuy hat Ende Juni 2012 seinen Vorschlag für die Weiterentwicklung der EU vorgelegt, dessen wesentlicher Baustein ein Durchgriffsrecht der EU auf die Haushalte der Mitgliedstaaten ist (Rompuy 2012).Zugespitzt heißt das: Weil die durch die Wettbewerbsordnung induzierte Kürzung der Sozialleistungen national auf zu großen Widerstand stößt, soll diese nun zentral durch die EU angeordnet werden. Diese Form einer autoritären Wirtschaftsregierung (ausführlich Fisahn 2011), über die eine strikte Austeritätspolitik verfolgt wird, bestimmt gegenwärtig den Entwicklungsweg der EU und ist die favorisierte „Krisenlösung“ der konservativen Kräfte unter Führung der deutschen Bundesregierung.

Eine derartige Form der Krisenlösung stößt erstens auf den Widerstand der Bevölkerung in den betroffenen Ländern, weshalb die Gefahr besteht, dass sie auch innenpolitisch durch autoritäre Maßnahmen abgesichert wird (Oberndorfer 2012: 68). Weil die Austeritätspolitik zweitens auch im Sinne der propagierten Ziele geradezu kontraproduktiv ist, wird es wahrscheinlich, dass sich andere 
Formen der Krisenbewältigung durchsetzen. Dabei besteht eine Variante im Erstarken zentrifugaler, national-chauvinistischer Tendenzen, die nicht nur am Zulauf entsprechend ausgerichteter politischer Organisationen von Front National und FPÖ über die Wahren Finnen bis de Wilders PVV abzulesen sind. Diese Tendenzen erreichen auch das politische Zentrum der EU, wenn man an die nur national orientierten Antworten auf die ökonomische Krise denkt, deren logische Folge die Wiedereinführung von Grenzkontrollen ist.

Nach der Wahl von Hollande zum französischen Präsidenten hat sich die europäische Sozialdemokratie auf den Weg zu einer eigenen Positionsbestimmung gemacht. Dabei zeichnet sich ab, dass die konservative Kürzungspolitik mitgetragen, aber ergänzt werden soll um eine Erhöhung der europäischen Strukturhilfen für die Krisenländer - was natürlich ein Widerspruch ist und nicht jeder Widerspruch ist Dialektik. Mit Blick auf die Finanzmärkte hat sich die Sozialdemokratie zur Forderung nach einer Transaktionssteuer durchgerungen, aber noch keineswegs zu einer Re-Regulierung der Finanzmärkte. Diese wäre allerdings mit dem sozialdemokratischen Modell des Neoliberalismus - Wettbewerbsfähigkeit durch Fordern und Fördern - vereinbar (Buckel u.a. 2012: 31).

Die europäische Linke hat sich zunächst auf die Abwehr der konservativen Austeritätspolitik verständigt und in dieser Auseinandersetzung konsolidiert und möglicherweise gestärkt, wenn man aus den Erfolgen von Syriza, der niederländischen SP oder von Jean-Luc Mélenchon eine Tendenz ableitet. Die Linke hat über die Regulierung der Finanzmärkte eine intensive Diskussion geführt und sinnvolle Vorschläge vorgelegt (Wahl 2009: 99), aber keineswegs eine konsistente Vorstellung einer europäischen Einigung jenseits des neoliberalen Modells. Ansätze für die Zukunft finden sich in Vorschlägen „Europa neu begründen“ oder eine verfassungsgebende Versammlung einzuberufen, die eine neue konstitutionelle Grundlage eines vereinten Europas jenseits des Wettbewerbsmodells entwickelt. Ein mittelfristiges Konzept müsste - wenn die vorstehende Analyse zutrifft - darauf drängen, erstens die Liberalisierungspflicht für den Kapitalverkehr aufzuheben und umzudrehen und zweitens durch Strukturveränderungen das Steuer- und Sozialdumping zu beenden, indem Vorschläge für eine intelligente Harmonisierung sowie eine eigenständige Finanzstruktur der EU mit der Möglichkeit, Konjunktur- und Sozialpolitik zu betreiben, entwickelt werden. Carlo Schmid hat schon bei der Diskussion um die EGKS auf den Konstruktionsfehler hingewiesen, der im Verlauf der europäischen Entwicklung nicht behoben, sondern verschärft wurde: „Wenn eine supranationale Behörde, oder sagen wir 'Autorität' funktionieren soll - wenn man das will, dann müsste die hohe Behörde umfassendere Verantwortung und Kompetenzen haben, als die hohe Behörde des Schumanplans sie hat. Sie müsste nämlich verpflichtet sein und dieser Verpflichtung müssten korrespondierende Befugnisse entsprechen 
-, für die sozialen und finanziellen Folgen ihrer wirtschaftlichen Anordnungen aufzukommen, mit Mitteln, die sie beschaffen muss" (Schmid,1951: 6512).

\section{Literatur}

Abendroth, W. (1979): Über den Zusammenhang von Grundrechtssystem und Demokratie, in: Perels, J. (Hrsg.), Grundrechte als Fundament der Demokratie, Frankfurt: 249 ff

Adenauer, K. (1951): Verhandlungen des Deutschen Bundestages I. Wahlperiode 1949, Stenografische Berichte Band 8.

Bieling, H.-J. (2003): Die neue europäische Ökonomie, in: Beckmann, M./Bieling, H.-J./Deppe, F. (Hg.), Eurokapitalismus und globale politische Ökonomie, Hamburg: $41 \mathrm{ff}$.

Borchardt, K.-D. (2002): Die rechtlichen Grundlagen der europäischen Union, Heidelberg.

Bsirske/Buntenbach/Hickel/Lehndorff/Urban (2012): Europa neu begründen, http://www. europa-neu-begruenden.de.

Buckel, S,/Georgi, F./Kannankulam, J./Wissel, J. (2012): Kräfteverhältnisse in der europäischen Krise, in: „Forschungsgruppe „Staatsprojekt Europa“(Hg.), Die EU in der Krise, Münster: 11.ff

Canfora, L. (2006): Eine kurze Geschichte der Demokratie Von Athen bis zur EU.

Coudenhove-Kalergi, R. (1923): Das paneuropäische Manifest, in: Schäfer, A.: Edition Europa, http://varzil.de/VV/INHALT.PDF: $19 \mathrm{ff}$.

Erhard, L. (1955): Gedanken von Ludwig Erhard zum Problem der Kooperation oder der Integration in Europa, http://www.cvce.eu/viewer/-/content.

EU Kommission (1999): Aktionsplan für Finanzbinnenmarkt, http://europa.eu/rapid/pressReleasesAction.do?

- (2008): Mitteilung der Kommission an den Europäischen Rat, Europäisches Konjunkturprogramm, vom 26.11.2008.

- (2011): Makroökonomischer Bericht, vom 23.11.2011, KOM(2011) 815, VOL. 3/5 - ANHANG II

Eurostat (2010): Pressemitteilung vom 28.6.2010, http://epp.eurostat.ec.europa.eu/cache/

EWG (1985): Richtlinie 85/611/EWG des Rates vom 20. Dezember 1985 zur Koordinierung der Rechts-und Verwaltungsvorschriften betreffend bestimmte Organismen fürgemeinsame Anlagen in Wertpapieren (OGAW) und Richtlinie 2001/107/EG des Europäischen Parlaments und des Rates vom 21. Januar 2002 zur Änderung der Richtlinie 85/611/EWG usw.

Fisahn A. (2011): Re-Regulierung der Finanzmärkte nach der Kernschmelze im Finanzsektor? RLS-Papers $7 / 2011$.

- (2008): Herrschaft im Wandel, Köln.

- (2011): Die Europäische Union auf dem Weg in eine autoritäre Wirtschaftsregierung, in: Zeitschrift Marxistische Erneuerung, Heft 87, Sept. 2011: 12.

FMSF-G (2008): Gesetz zur Errichtung eines Finanzmarktstabilisierungsfonds vom 17.10.2008, ergänzt durch die Verordnung zur Durchführung des Finanzmarktstabilisierungsfondsgesetzes, das Gesetz zur Beschleunigung und Vereinfachung des Erwerbs von Anteilen an Risikopositionen von Unternehmen des Finanzsektors durch den Fonds „Finanzmarktstabilisierungsfonds - FMS“ sowie das Gesetz zur Fortentwicklung der Finanzmarktstabilisierung.

Gill, St. (1998): European Governance and New Constitutionalism, in: New Political Economy 3(1): 5-26.

Grahl, J. (2003): Finanzintegration und europäische Gesellschaft, in: Beckmann, M./Bieling, H.-J./Deppe, F. (Hg.), Eurokapitalismus und globale politische Ökonomie, Hamburg: 18 ff.

Groeben, H./Schwarze,J. (2003): Kommentar zu EUV und EGV, Baden-Baden.

Habermas, J. (1973): Legitimationsprobleme im Spätkapitalismus, Frankfurt/M.

Haltern, U. (2007): Europarecht, Tübingen. 
Huffschmid, J. (1999): Politische Ökonomie der Finanzmärkte, Hamburg.

- (2008): Das „Europäische Sozialmodell“ - Nebelkerze oder politisches Konfliktfeld?, in: Viotto/ Fisahn (Hrsg.), Europa am Scheideweg, Hamburg: $68 \mathrm{ff}$.

Lenin, W.I. (1970): Über die Losung der Vereinigten Staaten von Europa, in: ders. Werke Bd. 21, Berlin: $342 \mathrm{ff}$.

Marx K. (1850): Die Klassenkämpfe in Frankreich 1848 bis 1850, MEW Berlin 1978, Bd. 7: 9 ff. Müller, K-P. (2007), Gemeinsamer EU-Finanzmarkt: Ein Weg mit vielen Etappen, in: Die Bank, Nr. 04/März 2007.

Oberndorfer, L. (2011): Economic Governance rechtswidrig - Eine Krisenerzählung ohne Kompetenz, in: AK Wien, Infobrief EU \& international, Juni 2011.

- (2012): Hegemoniekrise in Europa, in: „Forschungsgruppe 'Staatsprojekt Europa' (Hg.), Die EU in der Krise, Münster, S. 49.

Pollak, J./Slomski, P. (2012): Das politische System der EU, Wien.

Ritschl, H. (1951): Der Schumanplan: Die neue Ruhrbehörde, in: Der Spiegel 50/1951,: 20 ff,

Rompuy H.v. (2012): Aufdem Wegzu einer echten Wirtschafts-und Währungsunion, Bericht des

Präsidenten des Europäischen Rates, Herman Van Rompuy, 26. Juni 2012, EUCO 120/12.

Schmid, C. (1951): Verhandlungen des Deutschen Bundestages I. Wahlperiode 1949, Stenografische Berichte Band 8.

Schmidt, S. (1977): Kapitalverkehrskontrollen und ibre Wirkung, Hamburg.

Spinelli/Rossi (1941): Manifest von Ventotene, http://www.jef.de/index.php?id=1668.

Stamm, Ch. (2011): Die SPD unter Kurt Schumacher und Erich Ollenhauer 1946 bis 1963, Bonn.

Trotzki, L. (1923): Über die Aktualität der Parole „Vereinigte Staaten von Europa“, in: Prawda Nr.144, 30 Juni 1923, http://www.marxists.org/deutsch/archiv/trotzki/1923/06/vse.htm.

Wahl, P. (2009). Finanzmarktreform - von oben oder von unten, in: Sauer/Ötsch/Wahl (Hg.), Das Casino schließen, Hamburg; $87 \mathrm{ff}$.

Weidenfeld, W. (2011): Die europäische Union, Heidelberg.

Weniger, L. (1988): Kapitalverkehrskontrollen im Europäischen Währungssystem, Frankfurt/Main.

Zeise. L.: Geld - der vertrackte Kern des Kapitalismus, Köln 2010. 